\title{
Polystoma galamensis \\ (Monogenea) parasite de Rana galamensis \\ Duméril et Bibron, 1841, au Togo
}

\author{
par L. EUZET, R. BOURGAT et M.-L. SALAMI-CADOUX \\ Laboratoire de Parasitologie comparée, Université des Sciences et Techniques du Languedoc \\ Place E-Bataillon, F 34060 Montpellier Cedex, \\ et Laboratoire de Zoologie, Université de Benin, B.P. 1515, Lomé, Togo
}

\section{Résumé}

Description de Polystoma galamensis Monogène nouveau de la vessie urinaire de Rana galamensis au Togo. Ce parasite se caractérise par la faible valeur du rapport hapteur-corps, la taille importante des hamulis et le développement des caecums axiaux avec possibilité d'anastomose.

\section{Summary}

Polystoma galamensis (Monogenea) a parasite of Rana galamensis Dumeril and Bibron, 1841 from Togo.

A description of Polystoma galamensis a new Monogenea of the urinary bladder of Rana galamensis in Togo. This parasite is characterized by the low ratio haptor-body, the important size of the hamuli and the development of the axial coeca with a possibility of anastomosis.

Dans le cadre des recherches que nous avons entreprises sur les Helminthes parasites d'Amphibiens au Togo, nous avons découvert dans la vessie urinaire de Rana galamensis Duméril et Bibron, 1841 un Monogène Polystomatidae. La morphologie de ce parasite nous permet de le décrire comme une espèce nouvelle. L'hôte est commun dans la savane arborée de Klouto, située à une centaine de kilomètres au nord 
de Lomé. La répartition géographique connue de cet Anoure va du Sénégal à l'Ouganda et à la Tanzanie.

Nous avons examiné 57 Rana galamensis. Les dix récoltées aux environs immédiats de Lomé ne présentaient pas de Monogènes. Parmi les quarante-sept capturées à Klouto, dix étaient parasitées respectivement par 1, 1, 1, 1, 2, 2, 2, 4, 5 et 11 Polystomes, tous ovigères.

Nous en donnons ci-après la description.

\section{Polystoma galamensis n. sp.}

Hôte: Rana galamensis Duméril et Bibron, 1841.

HABITAT: Vessie urinaire.

Localité: Klouto (Togo).

MATÉRIEL ÉTUdí́ : 20 individus adultes colorés et montés in toto.

Les exemplaires étudiés mesurent de 8 à $10 \mathrm{~mm}$ de longueur totale. La largeur au niveau des vagins varie de 1,9 à $2,2 \mathrm{~mm}$, la largeur maximum en avant du hapteur de 2,6 à 3,2 mm. Le hapteur, sensiblement plus large que le corps, mesure de 1,7 à $1,9 \mathrm{~mm}$ de longueur, ce qui représente un peu plus du cinquième de la longueur totale. La place modeste occupée par le hapteur donne à l'animal une allure élancée (fig. 1).

Les ventouses subégales ont de 550 à $650 \mu$ de diamètre. Les hamuli, très développés, mesurent de 650 à $700 \mu$ de longueur. Le manche et la garde élargis sont séparés par une encoche irrégulière; l'oncosité dorsale de la lame est présente. Le manche subrectiligne est toujours plus long que la garde (fig. 2 et 3).

Chez quelques individus, nous avons pu observer les crochetons larvaires. Les crochetons postérieurs mesurent $35 \mu$ de longueur, les postéro-latéraux $12 \mu$. Les crochetons n'existent pas au centre des ventouses et les antérieurs sont masqués par le développement du tube digestif.

L'entonnoir buccal a de 550 à $600 \mu$ de largeur ; il donne accès à un pharynx piriforme qui mesure de 320 à $400 \mu$ de longueur et de 300 à $400 \mu$ de diamètre.

Les branches de l'appareil digestif présentent des caecums latéraux très courts. Les caecums axiaux, dépassant un peu le plan médian sagittal, peuvent réaliser des anastomoses transversales préhaptoriales. Sur 20 individus étudiés, 4 présentent une teile anastomose (fig. 4). Les deux branches digestives se réunissent toujours à la partie postérieure en une large anastomose haptoriale peu digitée.

L'anatomie du système génital est bâtie sur le même type que celle de Polystoma integerrimum.

Nous avons compté huit épines en couronne autour de l'ouverture génitale mâle. Ces épines, unies par leur base triangulaire, mesurent environ $35 \mu$ de longueur.

L'ovaire, à partie antérieure rétrécie et postérieure renflée, mesure $1 \mathrm{~mm}$ de longueur et $0,5 \mathrm{~mm}$ de diamètre. Chez nos exemplaires, il est situé 13 fois sur le côté droit 

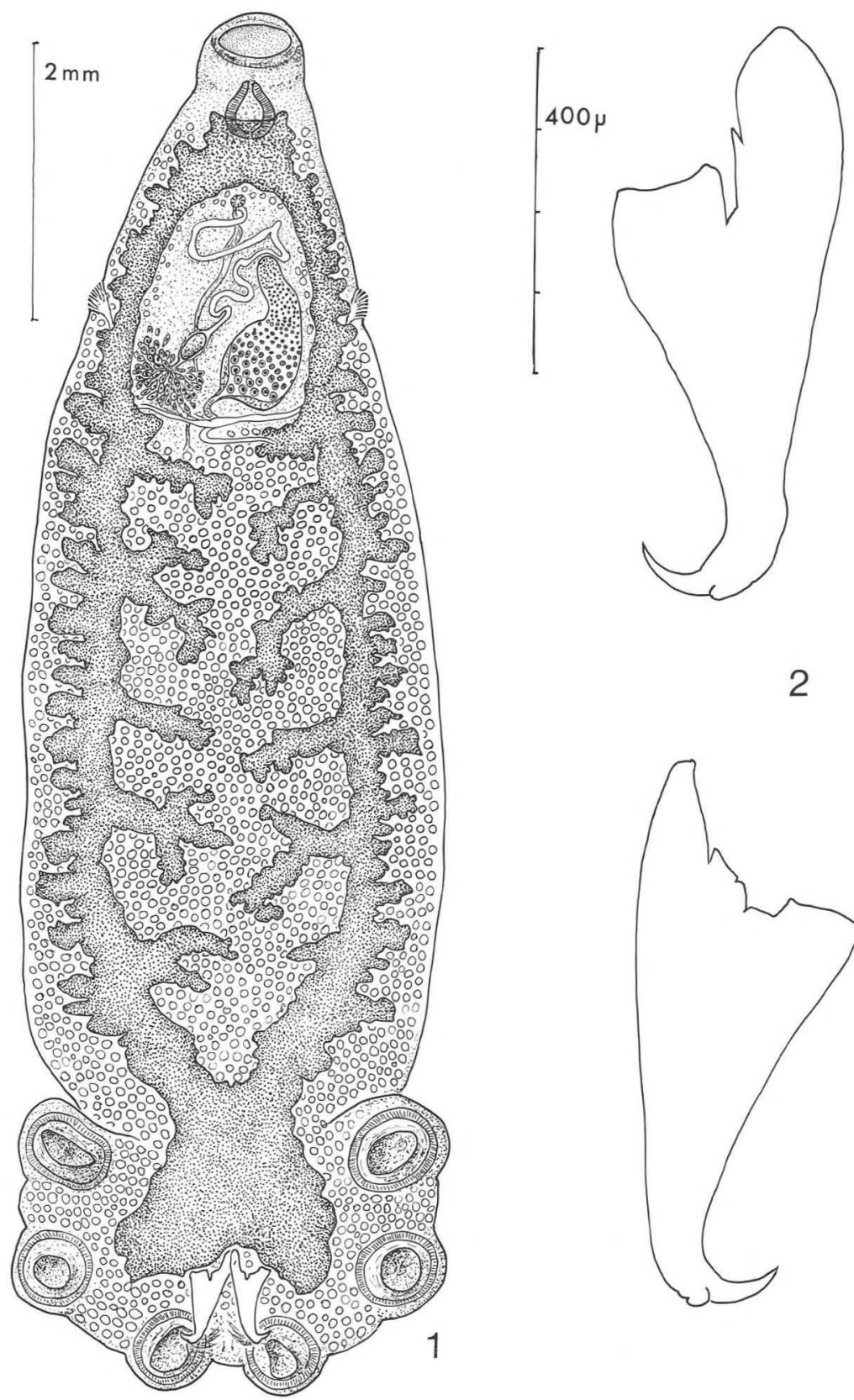

2

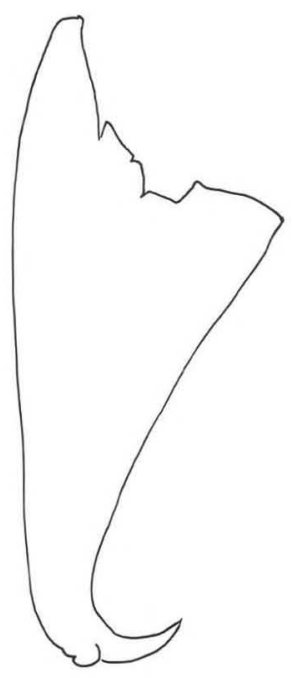

Polystoma galamensis n. sp.

FIG. 1. - Vue ventrale. Les glandes vitellogènes ventrales et le testicule ne sont pas représentés.

FIG. 2. - Hamuli.

Annales de Parasitologie humaine et comparée (Paris), t. 49, $\mathrm{n}^{\circ} 1$ 
du corps, 7 fois sur le côté gauche. Les deux vagins s'ouvrent latéralement au niveau du milieu de l'ovaire.

Sur les 20 individus étudiés, 17 avaient un œuf dans l'utérus, un deux, un autre trois, le dernier sept. Chez les Polystomes avec un œuf utérin, celui-ci renferme, chez six au moins, une larve entièrement formée avec les crochetons bien visibles.

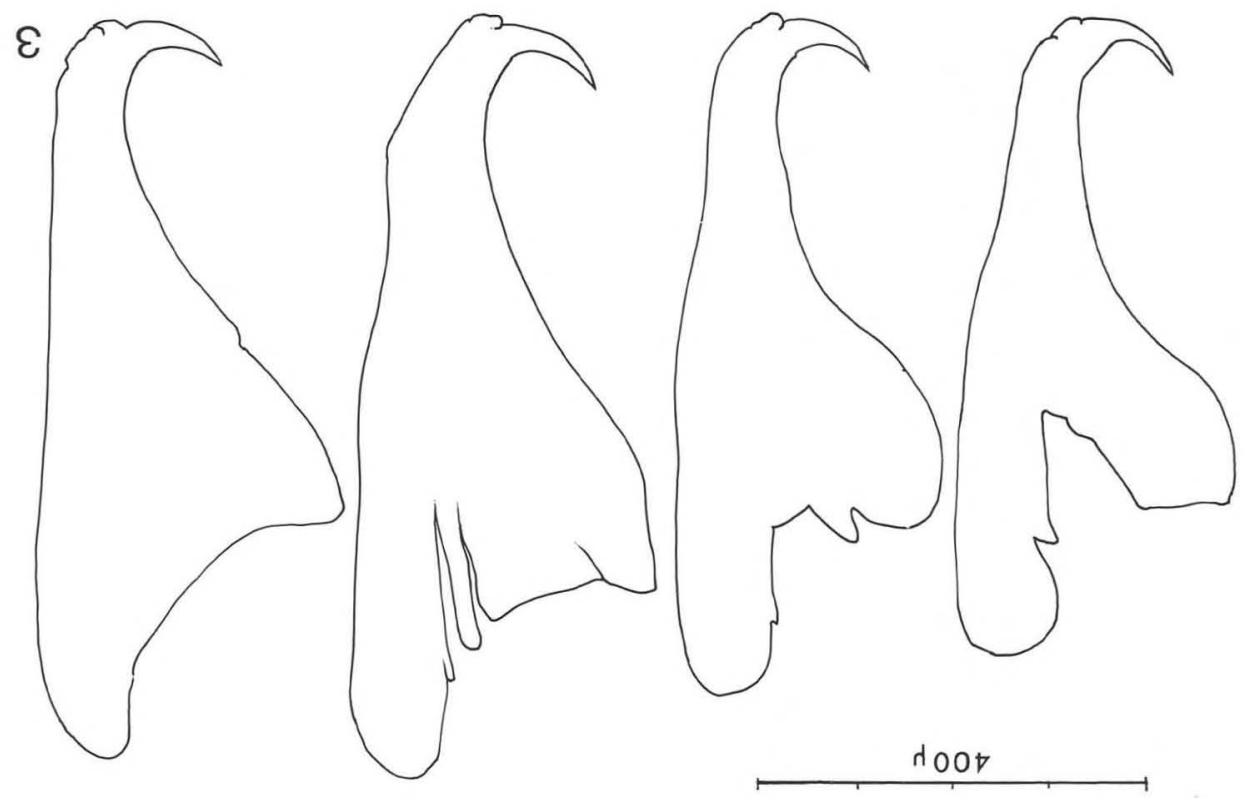

FIG. 3. - Polystoma galamensis n. sp. Variations de la morphologie des Hamuli.

Cette observation permet de penser que le cycle interne découvert et décrit par Combes (1968) chez $P$. integerrimum et $P$. pelobatis en Europe se produit également chez Polystoma galamensis au Togo. La larve, ainsi développée dans le dernier œuf utérin d'une période de reproduction, est capable de sortir de l'œuf dans la vessie, de se fixer à la paroi vésicale et de multiplier ainsi le parasite sur place.

Combes, Bourgat, Salami (1973) ont démontré que ce cycle interne, avec des modalités différentes, joue un rôle capital dans la dynamique de la population d'Eupolystoma alluaudi chez Bufo regularis au Togo.

Les œufs utérins mesurent de $140 \times 190 \mu$ à $150 \times 220 \mu$.

Tel que nous venons de le décrire, ce Polystome se caractérise par:

- sa grande taille, 
- la faible valeur du rapport hapteur-corps,

- les hamuli exceptionnellement grands,

- l'appareil digestif à anastomoses préhaptoriales peu fréquentes.

Cet ensemble de caractères permet d'affirmer que nous avons affaire à une espèce nouvelle que nous proposons de nommer Polystoma galamensis n. sp.
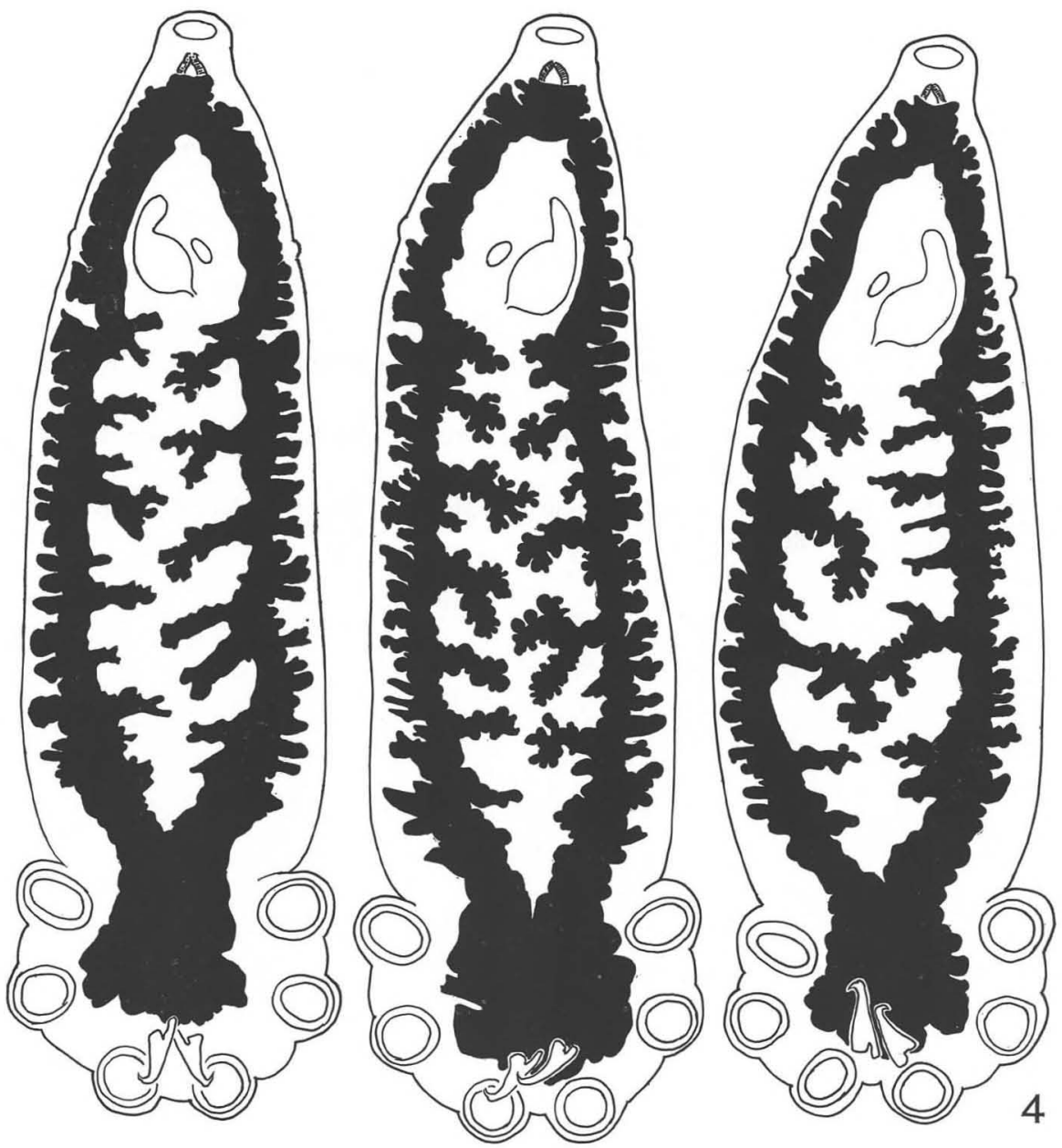

FIG. 4. - Polystoma galamensis n. sp. Anatomie du système digestif sans et avec anatomose transverse préhaptoriale. 


\section{Bibliographie}

Combes (C.), 1968. - Biologie, écologie des cycles et biogéographie des Digènes et Monogènes d'Amphibiens dans l'Est des Pyrénées. Mem. Mus. Natn. Hist. Nat. Paris, Ser. A, Zool., 51.

—, Bourgat (R.) et Salami-Cadoux (M.-L.). 1973. - Découverte d'un cycle interne direct intervenant comme mode habituel dans la reproduction d'un Plathelminthe. C.R. Acad. Sci. (Paris), 276, Ser. D, 2005-2006.

Euzet (L.), Combes (C.) et Knoepffler (L.-Ph.), 1966. — Parasites d'Amphibiens du Gabon Polystomatidae (Monogenea). Considérations sur la répartition géographique des Polystomatidae africains et malgaches actuellement connus. Biologica Gabonica, II (3), 215-233.

—, — et —, 1969. — Parasites d'Amphibiens de Côte-d'Ivoire et du Libéria, Polystomatidae (Monogenea). Biologica Gabonica, 5 (3), 217-221.

Gallien (L.), 1956. - Deux espèces nouvelles de Polystomes africains. Bull. Soc. Zool. France, 81 (5/6), 369-374.

Mafder (A.-M.), Euzet (L.) et Combes (C.), 1970. — Espèces nouvelles du genre Polystoma (Monogenea) en Afrique occidentale. Z. Parasitkde, 35, 140-155.

Meskal (F. S.), 1970. - Trematodes of Anurans from Ethiopia. Arb. Univ. Bergen. Mat. Naturv., série $\mathrm{n}^{\circ} 1,1-73$.

Szidat (L.), 1932. - Parasiten aus Liberia und Frenzosische Guinea. II Teil : Trematoden. Z. Parasitkde, 4 (2-3), 506-521.

Yamaguti (S.), 1963. - Systema helminthum. IV. Monogenea and Aspidocotylea. Interscience Publ., New York. 